\title{
Article \\ Adaptive Observer-Based Grid-Synchronization and Sequence Extraction Techniques for Renewable Energy Systems: A Comparative Analysis ${ }^{\dagger}$
}

\author{
Hafiz Ahmed 1,*(D) and Mohamed Benbouzid 2,3 (D) \\ 1 School of Engineering and the Built Environment, Birmingham City University, Birmingham B4 7XG, UK \\ 2 UMR CNRS 6027 IRDL, University of Brest, 29238 Brest, France; mohamed.benbouzid@univ-brest.fr \\ 3 Logistics Engineering College, Shanghai Maritime University, Shanghai 201306, China \\ * Correspondence: hafiz.h.ahmed@ieee.org \\ + This paper is an extended version of our paper published in the 46th Annual Conference of the IEEE \\ Industrial Electronics Society (IECON-2020), Singapore.
}

Citation: Ahmed, H.; Benbouzid, M. Adaptive Observer-Based

Grid-Synchronization and Sequence Extraction Techniques for Renewable Energy Systems: A Comparative Analysis. Appl. Sci. 2021, 11, 653. https://doi.org/10.3390/app11020653

Received: 9 November 2020 Accepted: 1 January 2021

Published: 11 January 2021

Publisher's Note: MDPI stays neutral with regard to jurisdictional clai$\mathrm{ms}$ in published maps and institutional affiliations.

Copyright: (C) 2021 by the authors. Licensee MDPI, Basel, Switzerland. This article is an open access article distributed under the terms and conditions of the Creative Commons Attribution (CC BY) license (https:// creativecommons.org/licenses/by/ $4.0 /)$.

\begin{abstract}
Various faults and disturbances often make the grid unbalanced and distorted. To make the grid-connected converters resilient to unbalanced and distorted grid, real-time estimation of grid voltage sequences are essential. Adaptive-observer based grid-synchronization techniques are very suitable for this purpose. In this paper, a comparative analysis of three recently proposed adaptive observer-based grid-synchronization technique are presented for frequency estimation and sequence extraction. The considered techniques are: global adaptive observer, gain normalized adaptive observer, and second-order generalized integrator type adaptive observer. Following a fair and systematic gain tuning process, the performances are verified through extensive numerical simulation and experiments. Experimental results demonstrate the merits and demerits of the individual techniques. Academic researchers and industrial practitioners will find the results useful for the selection of adaptive observer-based grid-synchronization and sequence extraction techniques.
\end{abstract}

Keywords: grid-synchronization; frequency-locked loop; sequence extraction; grid-connected renewable energy systems

\section{Introduction}

Renewable energy systems (RES) are the way forward towards achieving net-zero carbon emission target as mandated by many countries around the globe. This has contributed to a major push for ever increasing integration of RES into the existing electric power grid. This has attracted a lot of research attention into the control of grid-connected converters (GCC) [1-11]. Grid-connected converters can transfer maximum power to the grid only when the operation is in-phase with the grid voltage signal. Moreover, operation of GCC in unbalanced grid also require real-time extraction of grid voltage sequences. This motivated the researchers to devote significant attention to grid-synchronization based sequence extraction techniques [12-15].

Some of the recent and most popular grid synchronization techniques are: Kalman filter [16-18], demodulation [19,20], phase-locked loop (PLL) [21-26], adaptive observer [27-29], second-order generalized integrator (SOGI) [30-32], open-loop techniques [33], etc. Kalman filter [16] and least square-based techniques [34] typically work by considering a linear-parametric model of the grid voltage. Real-time matrix inversion are required for these techniques. This adds computational complexity for real-time low-cost embedded systems. Similar problems are to some extent true for the maximum likelihood estimator [18]. Demodulation [19] has several low-pass filters to tune, requires the use of trigonometric function, and can only be used for frequency estimation and not for sequence extraction.

Phase-locked loop (PLL) [21-26] has gained lot of attention for grid-synchronization application due to simplicity. Classical PLL generates double frequency oscillation in the 
presence of unbalanced grid. To avoid this issue, several solutions are proposed in the literature that require additional filtering. Moreover, tuning the PLL in the presence of additional filtering stage can be complicated. PLL is a closed-loop system where the stability is dependent on various parameters. Open-loop grid-synchronization technique [33] has a significant advantage in this regard as unconditional stability can be guaranteed. However, this type of technique often require calculating the derivative. Real-time estimation of signal derivatives are often challenging when the base signal is noisy.

Many nonlinear techniques are presented in the literature in recent times, such as adaptive observer [27-29,35], adaptive notch filter (ANF) [4], self-tuning filter (STF) [5], second-order generalized integrator (SOGI) [30-32], etc. Unlike PLL, these techniques perform the task of grid-synchronization through the help of frequency-locked loop. ANF, SOGI, and STF are similar in nature. All these techniques have band-pass filter property. SOGI has a limited complex conjugate closed-loop poles tuning range [36]. Adaptive observers [27-29] do not have any such limitation. This made adaptive observers-based technique a suitable choice for grid-synchronization in the literature.

Shah and Singh, in Reference [28], proposed the application of adaptive observer for grid-synchronization and sequence extraction. Using Lyapunov function and LaSalle's invariance principle, this observer provides global asymptotic stability of the state and parameter estimation error dynamics. Unfortunately, the frequency update law used in this work do not use any gain normalization. This makes the frequency estimation dynamics dependent on the grid voltage amplitude. This can slow down the convergence of the unknown frequency estimation when the grid voltages suffer significant sag. This issue has been resolved in Reference [27] by introducing gain normalized frequency estimation law. Both $[27,28]$ consider the grid voltage and its derivative as the state variables to obtain the dynamic model. SOGI-type adaptive observers as introduced in Reference $[29,36]$ use the same dynamical model as used by SOGI. In this case, quadrature signals are used as the state variables. This type of observer can further enhance the dynamic tuning range of SOGI.

In this paper, the focus is on comparative analysis of these recently proposed adaptive observers. Such a comparative comparison is not yet available in the literature. To this end, the observers are used for the purpose of grid-synchronization based sequence extraction for unbalanced and distorted grid. The comparison is facilitated by a systematic and fair gain tuning method. Extensive numerical simulations with adaptive notch filter-based frequency-locked loop [37-39] and experimental results are provided to highlight the advantages of the selected technique over the literature. Comprehensive and systemic presentation and extensive performance comparison of three recently proposed adaptive observer-based grid-synchronization techniques are the main contribution of this paper.

The rest of this paper is organized as follows: Problem formulation is given in Section 2, overview of the selected techniques are given in Section 3, numerical simulation and experimental performance comparison are given in Section 4, and, finally, Section 5 concludes this paper.

\section{Problem Formulation}

Unbalanced three-phase grid voltages comprise of positive $\left(^{+}\right)$, negative $\left(^{-}\right)$and zero $\left({ }^{0}\right)$ sequence components are given by: 


$$
\begin{aligned}
& U_{a}=\underbrace{A^{+} \sin \left(\omega t+\phi^{+}\right)}_{U_{a}^{+}}+\underbrace{A^{-} \sin \left(\omega t+\phi^{-}\right)}_{U_{a}^{-}}+\underbrace{A^{0} \sin \left(\omega t+\phi^{0}\right)}_{U_{a}^{0}}, \\
& U_{b}=\underbrace{A^{+} \sin \left(\omega t-\frac{2 \pi}{3}+\phi^{+}\right)}_{U_{b}^{+}}+\underbrace{A^{-} \sin \left(\omega t+\frac{2 \pi}{3}+\phi^{-}\right)}_{U_{b}^{-}}+\underbrace{A^{0} \sin \left(\omega t+\phi^{0}\right)}_{U_{b}^{0}}, \\
& U_{c}=\underbrace{A^{+} \sin \left(\omega t+\frac{2 \pi}{3}+\phi^{+}\right)}_{U_{c}^{+}}+\underbrace{A^{-} \sin \left(\omega t-\frac{2 \pi}{3}+\phi^{-}\right)}_{U_{c}^{-}}+\underbrace{A^{0} \sin \left(\omega t+\phi^{0}\right)}_{U_{c}^{0}},
\end{aligned}
$$

where the amplitude is denoted by $A$, the initial phase angle is denoted by $\phi$, and the angular frequency is denoted by $\omega$ with the nominal value being given by $\omega_{n}=100 \pi$. In this paper, the problem is to estimate the unknown angular frequency, $\omega$ and extract the positive $U^{+}=\left[\begin{array}{lll}U_{a}^{+} & U_{b}^{+} & U_{c}^{+}\end{array}\right]^{T}$, negative $U^{-}=\left[\begin{array}{lll}U_{a}^{-} & U_{b}^{-} & U_{c}^{-}\end{array}\right]^{T}$, and zero $U^{0}=\left[\begin{array}{lll}U_{a}^{0} & U_{b}^{0} & U_{c}^{0}\end{array}\right]^{T}$ sequence components from the measured grid voltages (1)-(3). For this purpose, let us consider the $90^{\circ}$ phase shifted version of the grid voltages (1)-(3):

$$
\begin{aligned}
& q U_{a}=\underbrace{A^{+} \cos \left(\omega t+\phi^{+}\right)}_{q U_{a}^{+}}+\underbrace{A^{-} \cos \left(\omega t+\phi^{-}\right)}_{q U_{a}^{-}}+\underbrace{A^{0} \cos \left(\omega t+\phi^{0}\right)}_{q U_{a}^{0}}, \\
& q U_{b}=\underbrace{A^{+} \cos \left(\omega t-\frac{2 \pi}{3}+\phi^{+}\right)}_{q U_{b}^{+}}+\underbrace{A^{-} \cos \left(\omega t+\frac{2 \pi}{3}+\phi^{-}\right)}_{q U_{b}^{-}}+\underbrace{A^{0} \cos \left(\omega t+\phi^{0}\right)}_{q U_{b}^{0}}, \\
& q U_{c}=\underbrace{A^{+} \cos \left(\omega t+\frac{2 \pi}{3}+\phi^{+}\right)}_{U_{c}^{+}}+\underbrace{A^{-} \cos \left(\omega t-\frac{2 \pi}{3}+\phi^{-}\right)}_{U_{c}^{-}}+\underbrace{A^{0} \cos \left(\omega t+\phi^{0}\right)}_{U_{c}^{0}} .
\end{aligned}
$$

From the grid voltages (1)-(3) and their quadrature version (4)-(6), the positive, negative and zero sequence components can be extracted by the following equations [39]:

$$
\begin{aligned}
& {\left[\begin{array}{l}
U_{a}^{+} \\
U_{b}^{+} \\
U_{c}^{+}
\end{array}\right]=\frac{1}{3}\left[\begin{array}{ccc}
1 & -\frac{1}{2} & -\frac{1}{2} \\
-\frac{1}{2} & 1 & -\frac{1}{2} \\
-\frac{1}{2} & -\frac{1}{2} & 1
\end{array}\right]\left[\begin{array}{l}
U_{a} \\
U_{b} \\
U_{c}
\end{array}\right]+\frac{1}{2 \sqrt{3}}\left[\begin{array}{ccc}
0 & 1 & -1 \\
-1 & 0 & 1 \\
1 & -1 & 0
\end{array}\right]\left[\begin{array}{l}
q U_{a} \\
q U_{b} \\
q U_{c}
\end{array}\right],} \\
& {\left[\begin{array}{l}
U_{a}^{-} \\
U_{b}^{-} \\
U_{c}^{-}
\end{array}\right]=\frac{1}{3}\left[\begin{array}{ccc}
1 & -\frac{1}{2} & -\frac{1}{2} \\
-\frac{1}{2} & 1 & -\frac{1}{2} \\
-\frac{1}{2} & -\frac{1}{2} & 1
\end{array}\right]\left[\begin{array}{l}
U_{a} \\
U_{b} \\
U_{c}
\end{array}\right]-\frac{1}{2 \sqrt{3}}\left[\begin{array}{ccc}
0 & 1 & -1 \\
-1 & 0 & 1 \\
1 & -1 & 0
\end{array}\right]\left[\begin{array}{l}
q U_{a} \\
q U_{b} \\
q U_{c}
\end{array}\right],} \\
& {\left[\begin{array}{l}
U_{a}^{0} \\
U_{b}^{0} \\
U_{c}^{0}
\end{array}\right]=\left\{\left[\begin{array}{lll}
1 & 0 & 0 \\
0 & 1 & 0 \\
0 & 0 & 1
\end{array}\right]-\frac{1}{3}\left[\begin{array}{ccc}
1 & -\frac{1}{2} & -\frac{1}{2} \\
-\frac{1}{2} & 1 & -\frac{1}{2} \\
-\frac{1}{2} & -\frac{1}{2} & 1
\end{array}\right]\right\}\left[\begin{array}{l}
U_{a} \\
U_{b} \\
U_{c}
\end{array}\right] .}
\end{aligned}
$$

From Equations (7)-(9), it is clear that to extract the positive, negative, and zero sequence components, quadrature signals are required. Adaptive observer-based gridsynchronization techniques will be used for this purpose. Details of the selected adaptiveobserver based techniques are given in the following section.

\section{Overview of the Selected Techniques}

\subsection{Global Adaptive Observer (GAO)}

This section briefly presents the globally asymptotically stable adaptive observer (GAO) as proposed in Reference [28,40]. 


\subsubsection{Development of Phase Voltage Dynamical Model}

Let us first consider the phase $a$. Models of phase $b$ and $c$ are the same as phase $a$. To develop the state-space model, let us first consider the phase voltage $U_{a}$ and its derivative as the following state variables:

$$
\begin{aligned}
& \xi_{1}=U_{a}=A^{+} \sin \left(\omega t+\phi^{+}\right)+A^{-} \sin \left(\omega t+\phi^{-}\right)+A^{0} \sin \left(\omega t+\phi^{0}\right) \\
& \xi_{2}=\dot{\xi}_{1}=\dot{U}_{a}=\omega\left\{A^{+} \cos \left(\omega t+\phi^{+}\right)+A^{-} \cos \left(\omega t+\phi^{-}\right)+A^{0} \cos \left(\omega t+\phi^{0}\right)\right\} .
\end{aligned}
$$

By considering the state vector, $\xi=\left[\begin{array}{ll}\xi_{1} & \xi_{2}\end{array}\right]^{T}$, the state-space model of the phase voltage dynamics can be written as:

$$
\begin{aligned}
\dot{\xi} & =\mathcal{A} \xi \\
U_{a} & =\mathcal{C} \xi
\end{aligned}
$$

where

$$
\mathcal{A}=\left[\begin{array}{cc}
0 & 1 \\
-\omega^{2} & 0
\end{array}\right], \mathcal{C}=\left[\begin{array}{ll}
1 & 0
\end{array}\right]
$$

State matrix $\mathcal{A}$ contains the unknown grid frequency $\omega$. To facilitate the frequency adaptation law development for $\omega$, let us rewrite the frequency in terms of the known nominal frequency as, $\omega^{2}=\eta \omega_{n}^{2}, \eta>0$. Observability matrix of system (10) and (11) is given by:

$$
\mathcal{O}=\left[\begin{array}{c}
\mathcal{C} \\
\mathcal{C} \mathcal{A}
\end{array}\right]=\left[\begin{array}{ll}
1 & 0 \\
0 & 1
\end{array}\right]
$$

Since the observability matrix $\mathcal{O}$ is of rank 2 which is the same as the rank of the state matrix $\mathcal{A}$, the system (10) and (11) is observable. Observer design for this system is described in the following section.

\subsubsection{Observer Design}

Let us the consider the following transformation for the state vector $\xi$ :

$$
\Xi=\mathcal{T} \xi,
$$

where $\Xi=\left[\begin{array}{ll}\Xi_{1} & \Xi_{2}\end{array}\right]^{T}$ is the transformed state vector and the non-singular matrix $\mathcal{T}$ is given by:

$$
\mathcal{T}=\frac{(1+\eta)^{-1}}{\omega_{n}^{2}}\left[\begin{array}{cc}
1 & -\frac{1}{\omega_{n}} \\
\eta \omega_{n} & 1
\end{array}\right]
$$

For the state vector $\Xi$, the transformed state and output matrices are given by:

$$
\mathcal{A}_{\Xi}=\mathcal{T} \mathcal{A} \mathcal{T}^{-1}=\mathcal{A}, \mathcal{C}_{\Xi}=\mathcal{C} \mathcal{T}^{-1} .
$$

Then, the transformed dynamical model for the phase voltage $U_{a}$ is given by:

$$
\begin{aligned}
\dot{\Xi} & =\mathcal{A}_{\Xi} \Xi, \\
U_{a} & =\mathcal{C}_{\Xi} \Xi .
\end{aligned}
$$

The following Luenberger observer can be designed for the system (14) and (15):

$$
\dot{\hat{\Xi}}=\hat{\mathcal{A}}_{\Xi} \hat{\Xi}+\mathcal{L}\left(U_{a}-\hat{U}_{a}\right)
$$

where^represents estimated value, $\hat{U}_{a}=\mathcal{C} \hat{\Xi}, \mathcal{L} \in \mathbb{R}^{2 \times 1}$ is the observer gain matrix, and

$$
\hat{\mathcal{A}}_{\Xi}=\left[\begin{array}{cc}
0 & 1 \\
-\hat{\eta} \omega_{n}^{2} & 0
\end{array}\right] .
$$


To study the convergence of the observer, let us consider the state and parameter estimation errors as:

$$
\begin{aligned}
& \tilde{\Xi}=\Xi-\hat{\Xi}, \\
& \tilde{\eta}=\eta-\hat{\eta} .
\end{aligned}
$$

Then, the closed-loop error dynamics for the system (14) and (15) and the observer (16) are given by:

$$
\begin{aligned}
\dot{\tilde{\Xi}} & =\dot{\Xi}-\dot{\hat{\Xi}}, \\
& =\mathcal{A}_{\Xi} \Xi-\left\{\hat{\mathcal{A}}_{\Xi} \hat{\Xi}+\mathcal{L}(\mathcal{C} \Xi-\mathcal{C} \hat{\Xi})\right\}, \\
& =\mathcal{A}_{\Xi} \Xi-\left\{\mathcal{A}_{\Xi} \hat{\Xi}-\mathcal{A}_{\Xi} \hat{\Xi}+\hat{\mathcal{A}}_{\Xi} \hat{\Xi}+\mathcal{L}\left(\mathcal{C}_{\Xi} \Xi-\mathcal{C}_{\Xi} \hat{\Xi}\right)\right\}, \\
& =\left(\mathcal{A}_{\Xi}-\mathcal{L} \mathcal{C}_{\Xi}\right)(\Xi-\hat{\Xi})+\left(\mathcal{A}_{\Xi}-\hat{\mathcal{A}}_{\Xi}\right) \hat{\Xi}, \\
& =\left(\mathcal{A}_{\Xi}-\mathcal{L} \mathcal{C}_{\Xi}\right) \tilde{\Xi}+\mathcal{R} \omega_{n}^{2} \tilde{\mu} \hat{\Xi}_{1},
\end{aligned}
$$

where $\mathcal{R}=\left[\begin{array}{ll}0 & 1\end{array}\right]^{T}$. For suitably designed observer gain matrix $\mathcal{L} \in \mathbb{R}^{2 \times 1}$, the matrix, $\mathcal{A}_{\Xi}-\mathcal{L C}_{\Xi}$ can be made Hurwitz, i.e., all the eigenvalues have strictly negative real parts. This makes the closed-loop error system strictly positive real (SPR) [41] and according to the SPR lemma, there exists a matrix $\mathcal{M}=\mathcal{M}^{T}>0$, such that

$$
\begin{aligned}
\left(\mathcal{A}_{\Xi}-\mathcal{L C}_{\Xi}\right)^{T} \mathcal{M}+M\left(\mathcal{A}_{\Xi}-\mathcal{L C}_{\Xi}\right) & =-\mathcal{Q}, \mathcal{Q}=\mathcal{Q}^{T}>0, \\
\mathcal{M R} & =\mathcal{C}_{\Xi}^{T} .
\end{aligned}
$$

The following Lyapunov function is considered for system (17):

$$
V(\tilde{\Xi}, \tilde{\eta})=\tilde{\Xi}^{T} \mathcal{M} \tilde{\Xi}+\gamma^{-1} \tilde{\eta}^{2}, \gamma>0 .
$$

Then, the derivative of the Lyapunov function becomes:

$$
\dot{V}=-\tilde{\Xi}^{T} \tilde{\Xi}+2\left(U_{a}-\hat{U}_{a}\right) \tilde{\eta} \omega_{n}^{2} \hat{\Xi}_{1}-2 \gamma^{-1} \tilde{\eta} \dot{\hat{\eta}} .
$$

If the frequency update law is chosen as:

$$
\dot{\hat{\eta}}=\gamma \omega_{n}^{2}\left(U_{a}-\hat{U}_{a}\right) \hat{\Xi}_{1} .
$$

Then, it becomes that $\dot{V} \leq 0$, which proves the Lyapunov stability of the estimation error dynamics. From the estimated transformed state vector $\hat{\Xi}$, the state vector $\xi$ can be found through the following equation:

$$
\hat{\zeta}=\mathcal{T}^{-1} \hat{\Xi},
$$

where

$$
\mathcal{T}^{-1}=\omega_{n}^{2}\left[\begin{array}{cc}
1 & \frac{1}{\omega_{n}} \\
-\hat{\eta} \omega_{n} & 1
\end{array}\right]
$$

The process described above can be applied in the same way to the grid phase voltages $U_{b}$ and $U_{c}$. As the frequency is same for all phases, only one frequency identification law is enough. From the signals $U_{a}, U_{b}$, and $U_{c}$ and their derivatives (i.e., quadrature signal) obtained through the observer (16), the sequences can be easily estimated through the Equations (7)-(9).

\subsection{Gain Normalized Adaptive Observer}

This section briefly presents the gain normalized adaptive observer (GNAO) as published in Reference [27]. This observer uses the same dynamic model as presented in Section 3.1.1. For further development, let us define the unknown frequency $\omega$ as, 
$\omega=\omega_{n}+\Delta \omega$, where $\Delta \omega$ is the frequency deviation. Let us consider the following state transformation:

$$
\Xi=\mathcal{T} \xi
$$

where

$$
\mathcal{T}=\frac{1}{2 \omega^{3}}\left[\begin{array}{cc}
\omega & -1 \\
\omega^{2} & \omega
\end{array}\right] .
$$

Then, the transformed state and output matrices are given by:

$$
\mathcal{A}_{\Xi}=\mathcal{T} \mathcal{A} \mathcal{T}^{-1}=\mathcal{A}, \mathcal{C}_{\Xi}=\mathcal{C} \mathcal{T}^{-1}=\left[\begin{array}{ll}
\omega^{2} & \omega
\end{array}\right] .
$$

Then, the following Luenberger type linear observer is designed:

$$
\dot{\hat{\Xi}}=\hat{\mathcal{A}}_{\Xi} \hat{\Xi}+\mathcal{L}\left(U_{a}-\hat{U}_{a}\right),
$$

where $\mathcal{L}=\left[\begin{array}{ll}\mathcal{L}_{1} & \mathcal{L}_{2}\end{array}\right]^{T}$ is the gain matrix for the observer and

$$
\hat{\mathcal{A}}_{\Xi}=\left[\begin{array}{cc}
0 & 1 \\
-\left(\omega_{n}+\Delta \hat{\omega}\right)^{2} & 0
\end{array}\right] .
$$

The frequency deviation estimation law is given by:

$$
\dot{\Delta \hat{\omega}}=-\frac{\gamma\left(\mathcal{L}_{1}+\mathcal{L}_{2}\right) \hat{\omega}^{3} \hat{\Xi}_{1}\left(U_{a}-\hat{U}_{a}\right)}{\sqrt{\frac{\left(\hat{\Xi}_{1} 2 \hat{\omega}^{3}\right)^{2}+\left(\hat{\Xi}_{2} 2 \hat{\omega}^{2}\right)^{2}}{2 \hat{\omega}^{2}}}}, \gamma>0 .
$$

The denominator term in the right hand side of Equation (27) scales the frequency deviation update law by the estimate of the grid voltage signal amplitude. This makes the adaptation law less sensitive to large voltage sag/swell. This can be very suitable to provide low voltage ride through capability if the observer is used inside the control module of any grid-connected converter.

Lyapunov function-based stability analysis as presented in Section 3.1.2 cannot be applied to the observer (25) due to the presence of gain normalization term in Equation (27). As such, only local stability results are presented in Reference [27]. From the estimated state vector $\hat{\Xi}$, the state vector $\hat{\xi}$ can be calculated through the following equation:

$$
\hat{\Theta}=\mathcal{T}^{-1} \hat{\xi},
$$

where

$$
\mathcal{T}^{-1}=\left[\begin{array}{cc}
\hat{\omega}^{2} & \hat{\omega} \\
-\hat{\omega}^{3} & \hat{\omega}^{2}
\end{array}\right]
$$

The same observer (25) can be applied to grid phase voltages $U_{b}$ and $U_{c}$. From the signals $U_{a}, U_{b}$, and $U_{c}$ and their derivatives (i.e., quadrature signal) obtained through the observer (25), the sequences can be easily estimated through the Equations (7)-(9).

\subsection{SOGI-Type Adaptive Observer}

This Section briefly presents the SOGI-type adaptive observer (SAO) result proposed in Reference [29]. Unlike the previous two observers, this observer uses a different dynamic model.

\subsubsection{Quadrature Signal-Based Dynamic Model Development}

For model development purposes, let us consider that the states are: 


$$
\begin{aligned}
& \xi_{1}=U_{a}^{\perp}=-A^{+} \cos \left(\omega t+\phi^{+}\right)-A^{-} \cos \left(\omega t+\phi^{-}\right)-A^{0} \cos \left(\omega t+\phi^{0}\right), \\
& \xi_{2}=U_{a}=A^{+} \sin \left(\omega t+\phi^{+}\right)+A^{-} \sin \left(\omega t+\phi^{-}\right)+A^{0} \sin \left(\omega t+\phi^{0}\right) .
\end{aligned}
$$

Then, the following can be obtained:

$$
\begin{aligned}
& \dot{\xi}_{1}=\xi_{2} \omega, \\
& \dot{\xi}_{2}=-\xi_{1} \omega .
\end{aligned}
$$

By considering the state vector, $\xi=\left[\begin{array}{ll}\xi_{1} & \xi_{2}\end{array}\right]^{T}$, the dynamic model for the grid phase voltage $U_{a}$ is given by:

$$
\begin{aligned}
\dot{\xi} & =\mathcal{A} \xi, \\
U_{a} & =\mathcal{C} \xi,
\end{aligned}
$$

where

$$
\mathcal{A}=\left[\begin{array}{cc}
0 & \omega \\
-\omega & 0
\end{array}\right], \mathcal{C}=\left[\begin{array}{ll}
0 & 1
\end{array}\right]
$$

Observability matrix of system (29) and (30) is given by:

$$
\mathcal{O}=\left[\begin{array}{c}
\mathcal{C} \\
\mathcal{C} \mathcal{A}
\end{array}\right]=\left[\begin{array}{cc}
0 & 1 \\
-\omega & 0
\end{array}\right]
$$

Since the observability matrix $\mathcal{O}$ is of rank 2 which is the same as the rank of the state matrix $\mathcal{A}$, the system (29) and (30) is observable. Observer design for this system is described in the following section.

\subsubsection{Observer Design}

Let us consider the following transformation:

$$
\Xi=\mathcal{T} \xi,
$$

where

$$
\mathcal{T}=\frac{1}{2 \omega}\left[\begin{array}{cc}
1 & 1 \\
-1 & 1
\end{array}\right]
$$

Then, the transformed state and output matrices are given by:

$$
\mathcal{A}_{\Xi}=\mathcal{T} \mathcal{A} \mathcal{T}^{-1}=\mathcal{A}, \mathcal{C}_{\Xi}=\mathcal{C} \mathcal{T}^{-1}=\left[\begin{array}{ll}
\omega & \omega
\end{array}\right] .
$$

Then, the transformed dynamic model for the phase voltage $U_{a}$ is given by:

$$
\begin{aligned}
\dot{\Xi} & =\mathcal{A}_{\Xi} \Xi, \\
U_{a} & =\mathcal{C}_{\Xi} \Xi .
\end{aligned}
$$

For model (33) and (34), the following Luenberger type linear observer is designed:

$$
\dot{\hat{\Theta}}=\hat{\mathcal{A}}_{\Xi} \hat{\Xi}+\mathcal{L}\left(U_{a}-\hat{U}_{a}\right),
$$

where $\mathcal{L}=\left[\begin{array}{ll}\mathcal{L}_{1} & \mathcal{L}_{2}\end{array}\right]^{T}$ is the gain matrix for the observer and

$$
\hat{\mathcal{A}}_{\Xi}=\left[\begin{array}{cc}
0 & \omega_{n}+\hat{\Delta \omega} \\
-\left(\omega_{n}+\Delta \hat{\omega}\right) & 0
\end{array}\right] .
$$


Moreover, the gain normalized frequency deviation estimation law is given by:

$$
\Delta \dot{\hat{\omega}}=-\frac{\gamma\left(\mathcal{L}_{1}+\mathcal{L}_{2}\right) \hat{\omega} \hat{\Xi}_{1}\left(U_{a}-\hat{U}_{a}\right)}{\hat{\Xi}^{T} \hat{\Xi}}, \gamma>0
$$

Similar to the gain normalized adaptive observer presented in Section 3.2, only local stability results are presented in Reference [29] due to the presence of the gain normalization term in Equation (37). From the estimated state vector $\hat{\underline{\Xi}}$, the state vector $\hat{\xi}$ can be calculated through the following equation:

$$
\hat{\xi}=\mathcal{T}^{-1} \hat{\underline{\Xi}},
$$

where

$$
\mathcal{T}^{-1}=\hat{\omega}\left[\begin{array}{cc}
1 & -1 \\
1 & 1
\end{array}\right]
$$

The same observer (35) can be applied to grid phase voltages $U_{b}$ and $U_{c}$. From the signals $U_{a}, U_{b}$, and $U_{c}$ and their derivatives (i.e., quadrature signal) obtained through the observer (35), the sequences can be easily estimated through the Equations (7)-(9).

\section{Results and Discussions}

In this section, comparative performance verification is considered. The selected observers are tuned by considering the closed-loop poles as: $-1.5 \omega_{n} \pm i \omega_{n}$. This corresponds to $\mathcal{L}=\left[\begin{array}{ll}0.0012 & 2.6250\end{array}\right]^{T}$ (GAO and GNAO) and $\mathcal{L}=\left[\begin{array}{ll}0.3750 & 2.6250\end{array}\right]^{T}$. A 2-cycle convergence criterion is used to tune the parameter $\gamma$ through trial and error. This corresponds to the value of $\gamma$ as 1000, 150 and 0.2 for GAO, GNAO, and SAO, respectively. With these gains, the selected techniques have the same dynamic response in the presence of frequency step change in the ideal grid voltage situation. FLL-based adaptive notch filter (ANF-FLL) [37-39] is selected as the comparison technique. The ANF gain is selected as $1 / \sqrt{2}$, while the FLL gain is selected as 18,000 , as suggested in Reference [39].

\subsection{Simulation Results}

The following test cases are considered in this section:

- Test-I: Balanced to unbalanced grid,

- Test-II: Voltage sag,

- Test-III: Phase step change, and

- Test-IV: DC offset.

Figure 1 shows the comparative simulation for Test-I. In this test, the negative and zero sequence voltage had a sudden step change of +0.1 p.u. and +0.05 p.u., while the positive sequence voltage had a step change of $-0.2 \mathrm{p}$.u. The frequency estimated by the adaptive observer-based techniques converged in 2-cycles, while ANF took 2.5 cycles with significantly higher overshoot. In addition, the positive, negative, and zero sequence amplitudes are estimated within $\approx 0.5$ cycle by the adaptive observer-based techniques, while it is more than $\approx 1$ cycle for ANF.

Voltage sag of -0.5 p.u. in balanced grid is considered in Test-II. Simulation results are given in Figure 2. As shown in Figure 2c, SAO and GNAO converged within 2 cycles, and GAO took 3 cycles, while, for ANF, it is almost 5 cycles. This shows that adaptive observer-based techniques are suitable for fast and accurate estimation of grid frequency in the presence of large voltage sag. In case of positive sequence amplitude estimation, half cycle convergence can be observed for the adaptive observer-based techniques, while it is $\approx 1$ cycle for the ANF-FLL.

A sudden jump of $-45^{\circ}$ phase angle in a balanced grid is considered in Test-III. Simulation results as shown in Figure 3 demonstrate that all the techniques are heavily affected by the sudden change in phase angle. However, adaptive observer-based techniques converged very fast within roughly 45 milliseconds, while ANF took more than 60 milliseco-nds. In addition, the peak overshoot for ANF was also $\approx+1.5 \mathrm{~Hz}$ higher than the adaptive observer-based techniques. This demonstrates the advantage of the adaptive 
observer-based techniques for challenging scenarios, such as sudden large change in phase angle.

Due to current transformer saturation, signal conditioning problem, etc., DC offset can often corrupt the measured voltages. This is considered in Test-IV. The considered DC offset values are +0.05 p.u., +0.1 p.u., and -0.05 p.u. in phase $a, b$, and c, respectively. Results in Figure $4 \mathrm{~b}$ show that ANF is very sensitive to DC offset. The frequency estimation ripple is between $48.2 \mathrm{~Hz}$ and $50.8 \mathrm{~Hz}$ for ANF. Among the adaptive observer-based techniques, GAO is the most sensitive with estimation ripple between $49 \mathrm{~Hz}$ and $50.78 \mathrm{~Hz}$. GNAO and SAO have similar estimation ripple and the amplitude is lower than ANF and GAO.

By considering the test results as shown in Figures 1-4, it can be claimed that adaptive observer-based techniques have performed better than ANF in almost every situations. As such ANF will not be considered for experimental validation test in Section 4.2. Among adaptive observer-based techniques, GAO under performed compared to GNAO and SAO. It is to be noted here that all the adaptive observer-based techniques have similar dynamic performance.

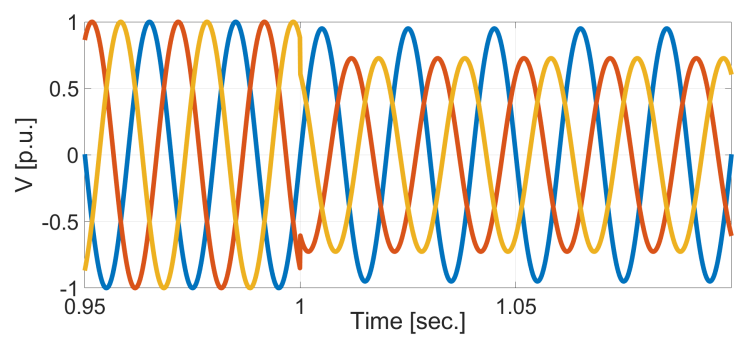

(a) Simulated three-phase grid voltage signal

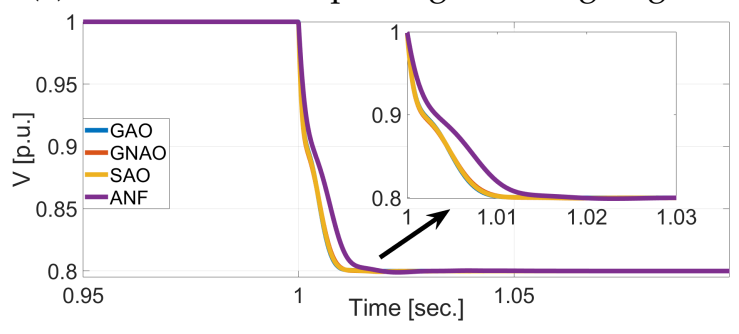

(c) Extracted positive sequence amplitudes

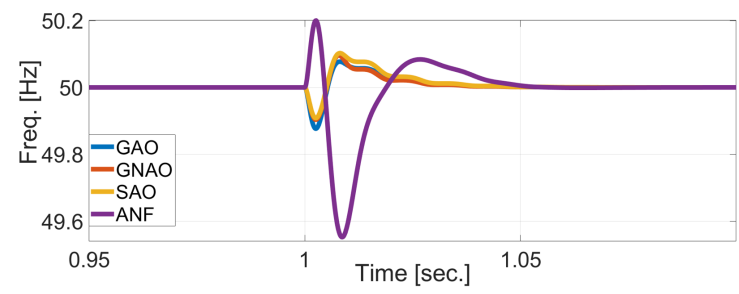

(b) Estimated frequencies

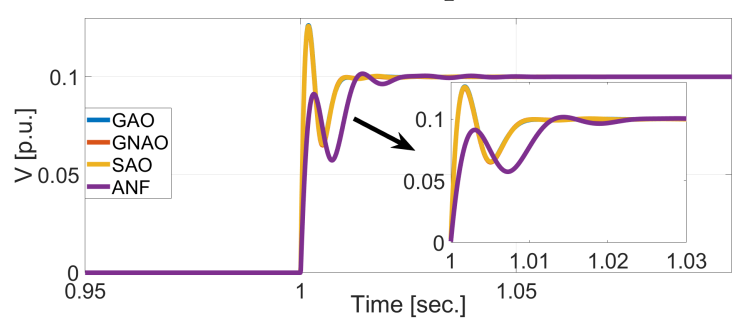

(d) Extracted negative sequence amplitudes

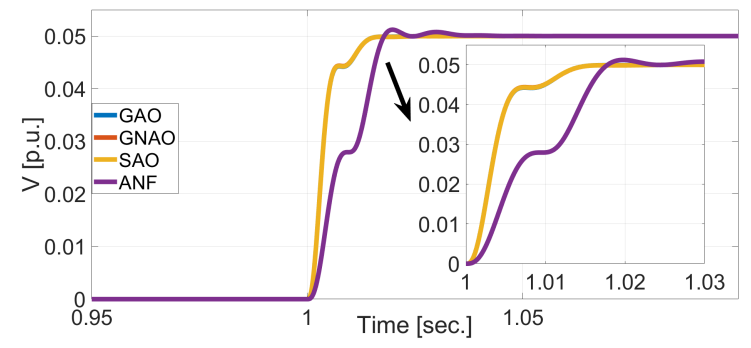

(e) Extracted zero sequence amplitudes

Figure 1. Numerical simulation results for Test-I. 


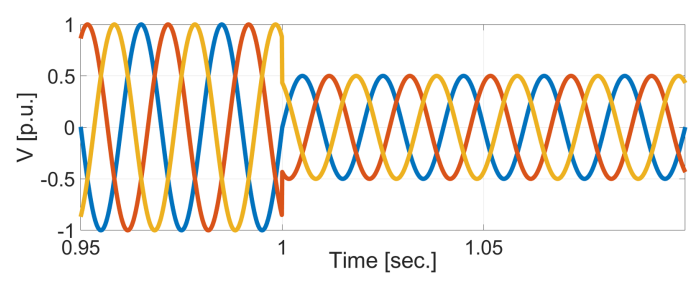

(a) Simulated three-phase grid voltage signal

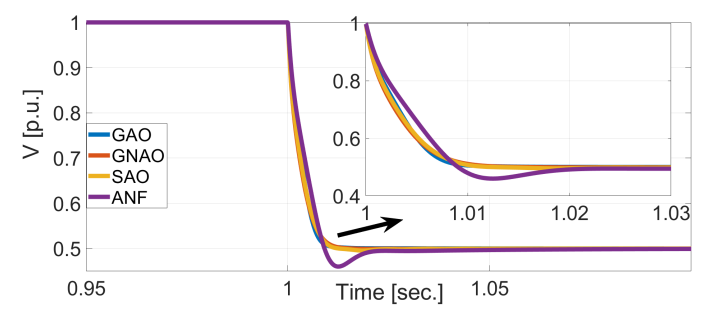

(b) Extracted positive sequence amplitudes

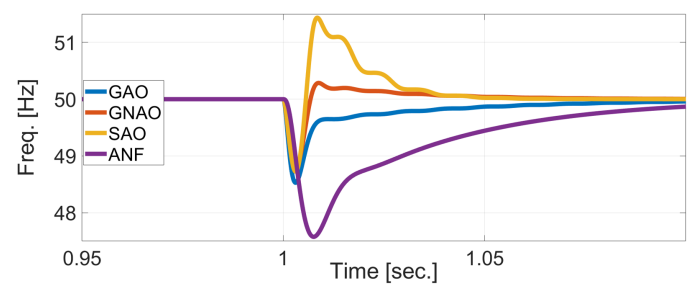

(c) Estimated frequencies

Figure 2. Numerical simulation results for Test-II.

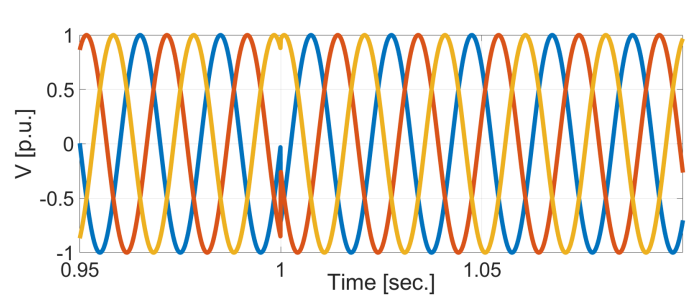

(a) Simulated three-phase grid voltage signal

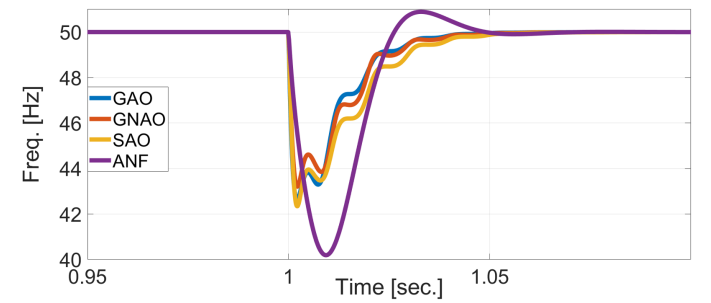

(b) Estimated frequencies

Figure 3. Numerical simulation results for Test-III.

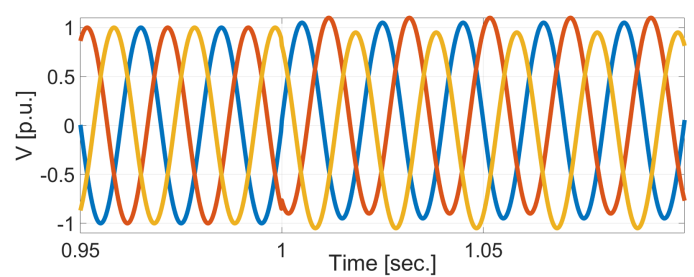

(a) Simulated three-phase grid voltage signal

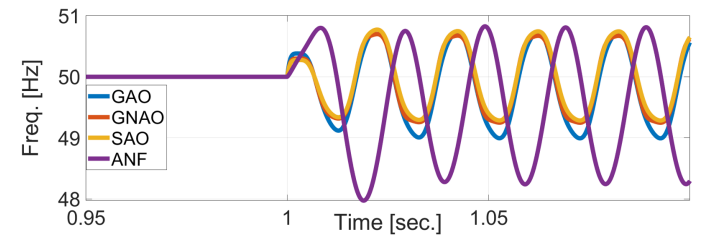

(b) Estimated frequencies

Figure 4. Numerical simulation results for Test-IV.

\subsection{Experimental Results}

For the experimental validation, dSPACE MicroLabBox is used to implement the adaptive observer-based techniques in real-time. An overview of the experimental setup is given in Figure 5.

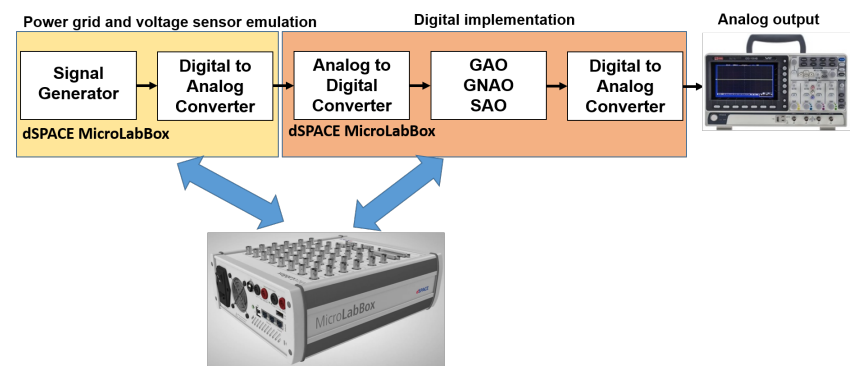

Figure 5. Experimental setup overview. 


\subsubsection{Unbalanced Grid}

In this test, initially the grid is balanced, i.e., no negative and zero sequence components. Suddenly, the positive sequence amplitude had a step change of -0.2 p.u. Moreover, negative sequence and positive sequence voltages of $0.2 \mathrm{p} . \mathrm{u}$. and $0.1 \mathrm{p} . \mathrm{u}$. are added into the grid. In addition, the phase angle of the positive, negative, and zero sequence components had a step change of $-22.5^{\circ},-22.5^{\circ}$, and $22.5^{\circ}$, respectively. Experimental results for this test are given in Figure 6. From the estimated frequencies, it can be seen that the three techniques have roughly similar dynamic performance and converged in $\approx 2$ cycles. This validates the gain tuning. GAO converged faster than the others, however, with the highest peak overshoot. Similarly, SAO had the lowest peak overshoot and the same time the slowest convergence time among the selected techniques. The sequence extraction performance of all the techniques are similar. The techniques extracted the positive, negative, and zero sequence components from the measured three-phase grid voltage in a fast and accurate manner.

\subsubsection{Unbalanced and Distorted Grid}

Grid distortion is often inevitable. So, this test considers unbalance and distortion at the same time. In this test, initially balanced grid is consider with 1.0 p.u. positive sequence component. After the fault, negative sequence of 0.1 p.u. and zero sequence of 0.05 p.u. are added. In addition, 5th and 13th-order positive sequence harmonics of 0.015 p.u. and 0.012 p.u. and 7th-order negative sequence harmonics of 0.017 p.u. are added. So, the negative sequence component has a distortion of $17 \%$ which is a highly challenging test scenario. Experimental results are given in Figure 7. Due to the presence of distortion in the voltage, the estimated frequencies also have estimation ripple. Similar to the previous test, GAO has the highest peak overshoot $(\approx 0.5 \mathrm{~Hz})$ and steady-state estimation ripple $(\approx 0.3 \mathrm{~Hz})$. SAO demonstrated the lowest peak overshoot $(\approx 0.3 \mathrm{~Hz})$ and steady-state estimation ripple $(\approx 0.1 \mathrm{~Hz})$.

Based on simulation and experimental results, a comparative summary of the adaptive observer-based techniques are presented in Table 1.

Table 1. Comparative analysis of the adaptive observer-based techniques.

\begin{tabular}{cccc}
\hline Characteristics & GAO & GNAO & SAO \\
\hline Dynamic Performance & Very Fast & Very Fast & Very Fast \\
\hline Voltage Sag Sensitivity & High & Low & Low \\
\hline Harmonic Disturbance & Good & Very Good & Very Good \\
\hline DC Offset Rejection & Good & Very Good & Very Good \\
\hline Computational Complexity & Medium & High & Medium \\
\hline
\end{tabular}




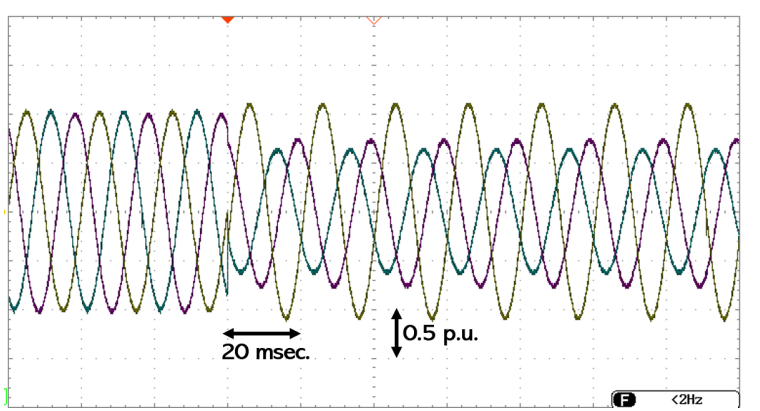

(a) Experimental three-phase grid voltage signal

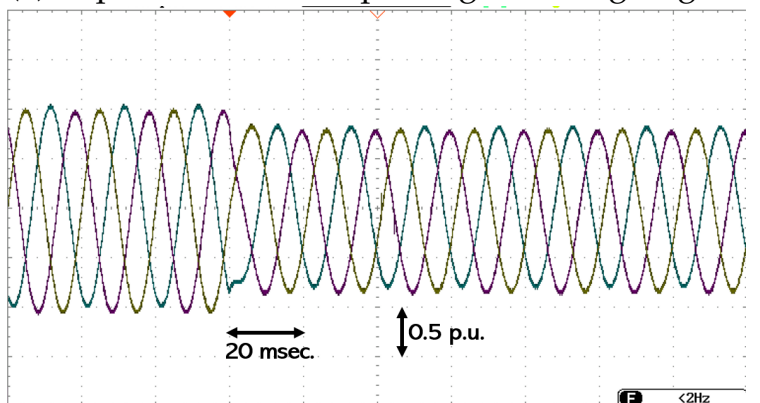

(c) Extracted positive sequence by GAO

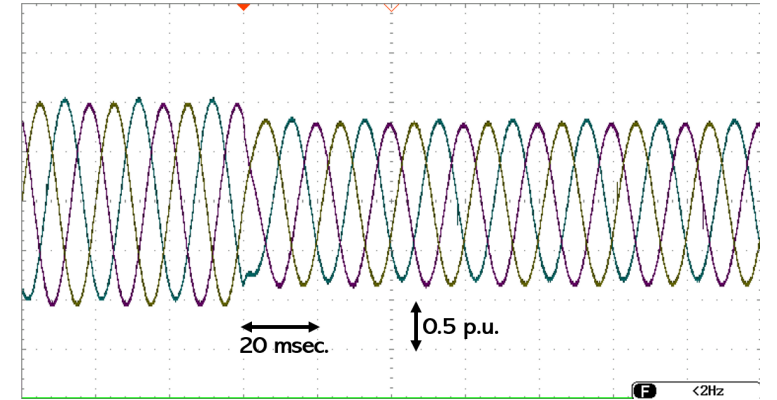

(e) Extracted positive sequence by $\mathrm{SAO}$

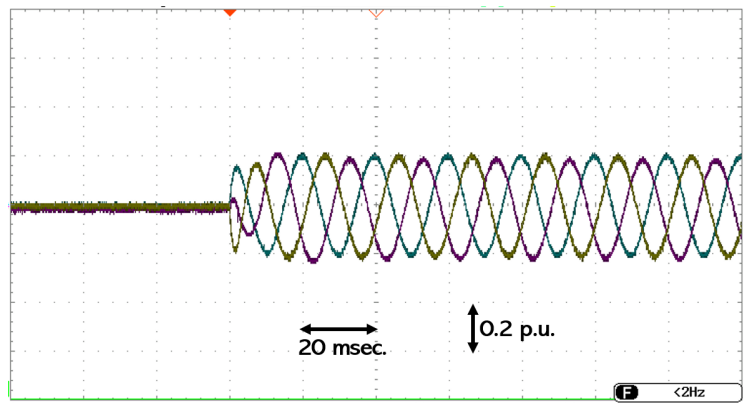

(g) Extracted negative sequence by GNAO

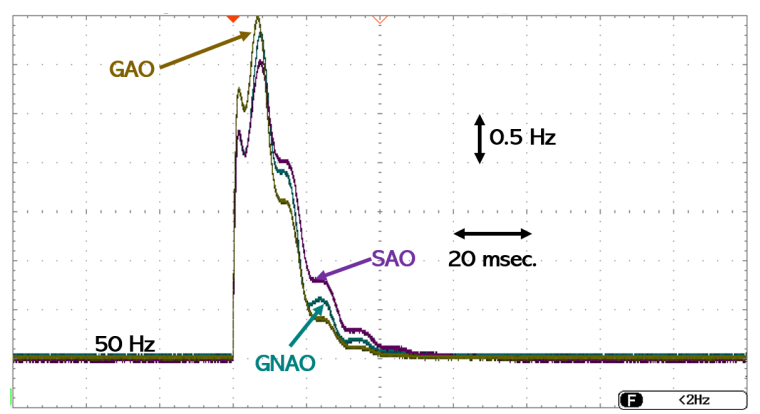

(b) Estimated frequencies

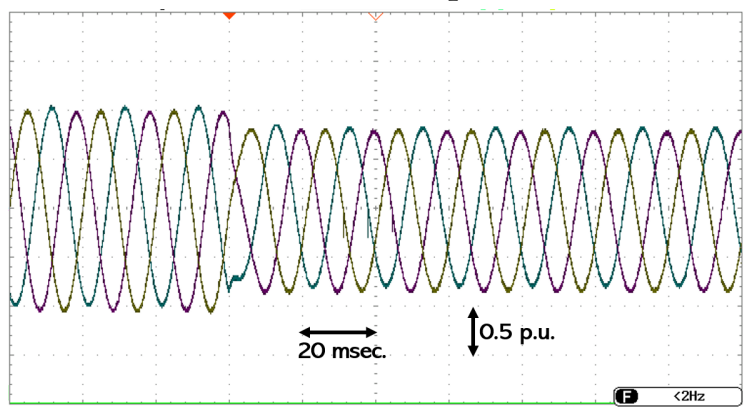

(d) Extracted positive sequence by GNAO

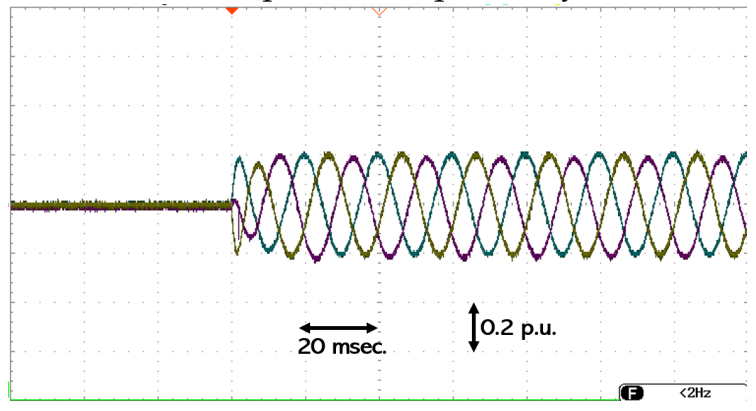

(f) Extracted negative sequence by GAO

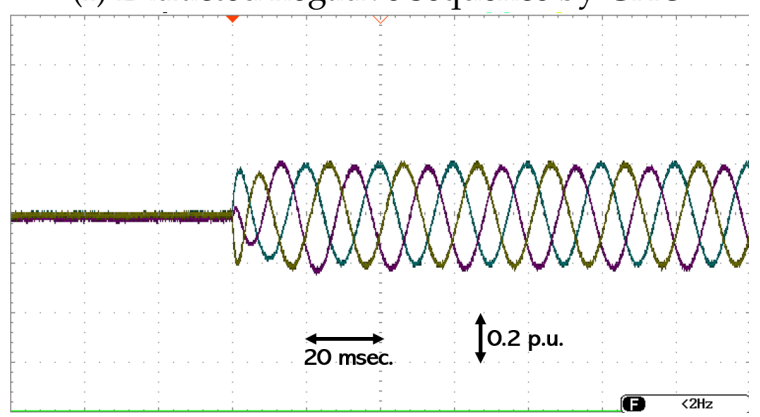

(h) Extracted negative sequence by $\mathrm{SAO}$

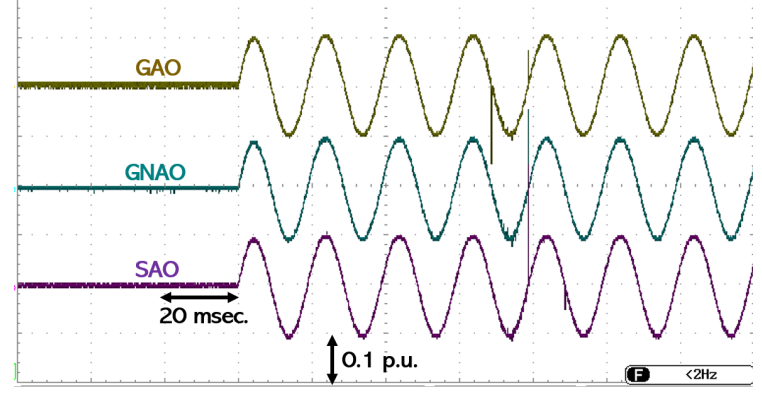

(i) Extracted zero sequence component by the selected techniques

Figure 6. Experimental results for balanced to unbalanced grid. 


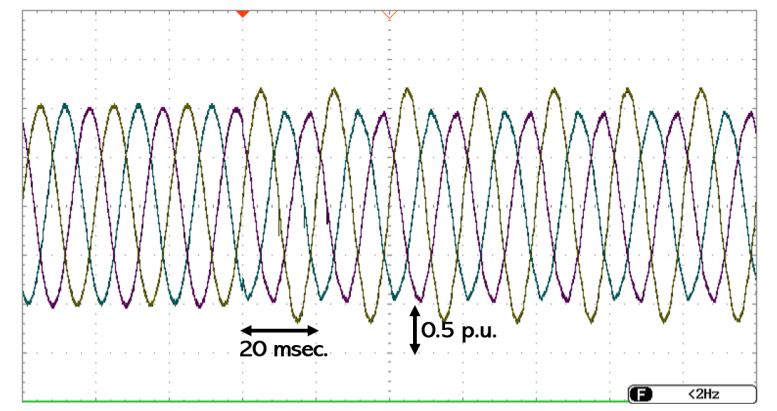

(a) Experimental three-phase grid voltage signal

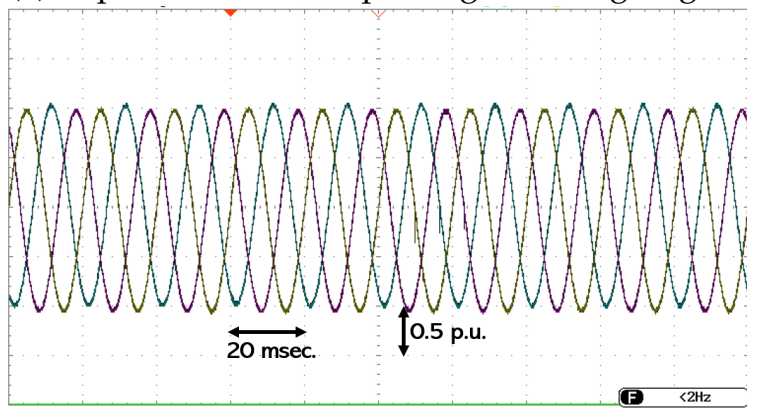

(c) Extracted positive sequence by GAO

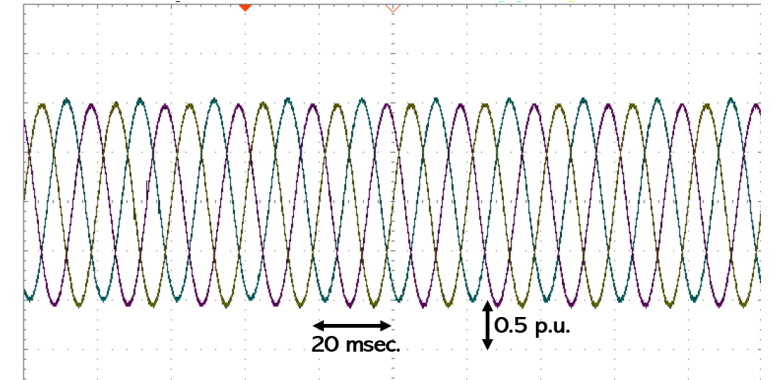

(e) Extracted positive sequence by $\mathrm{SAO}$

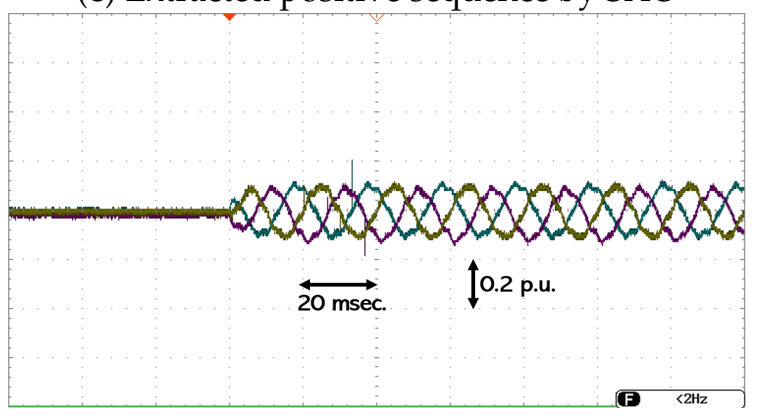

(g) Extracted negative sequence by GNAO

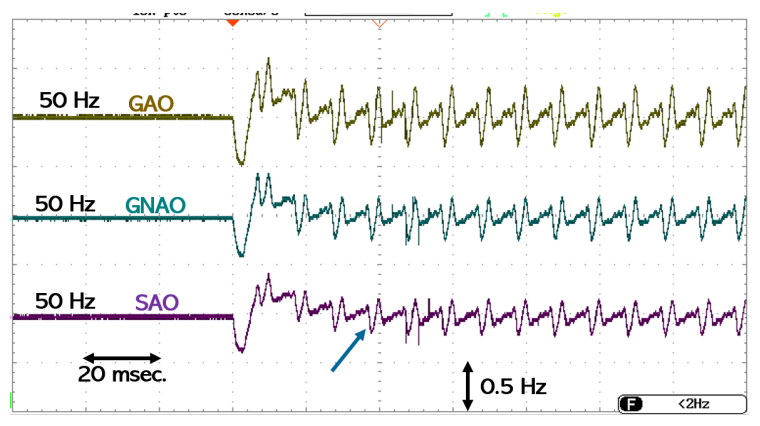

(b) Estimated frequencies

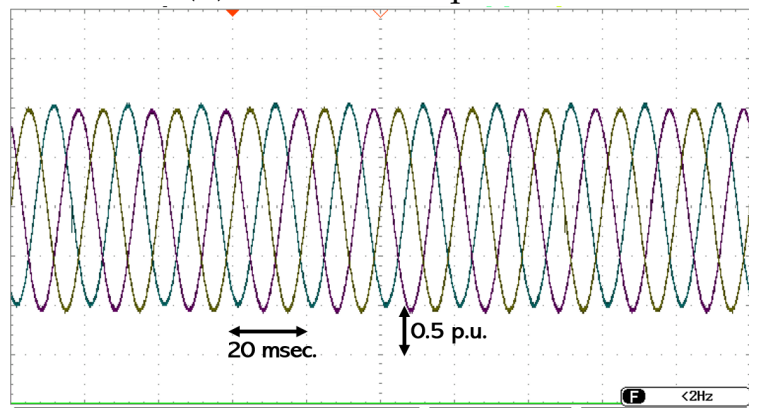

(d) Extracted positive sequence by GNAO

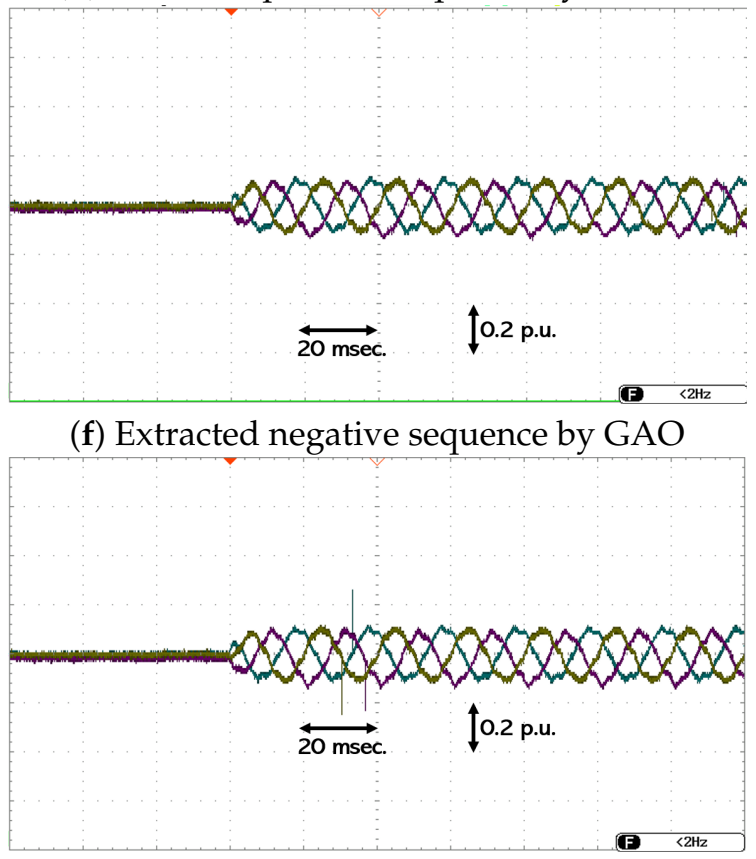

(h) Extracted negative sequence by SAO

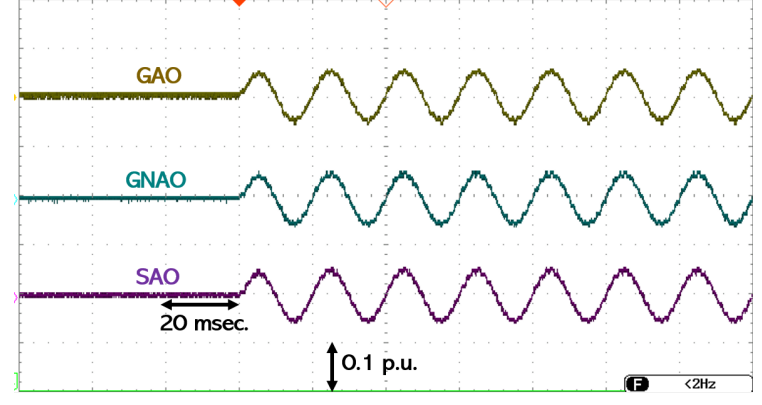

(i) Extracted zero sequence component by the selected techniques

Figure 7. Experimental results for unbalanced and distorted grid. 


\section{Conclusions}

Three recently proposed adaptive observer-based grid-synchronization techniques are presented in this paper. These techniques are used to extract the positive, negative, and zero sequence components of unbalanced and distorted three-phase grid voltage signal. A systematic gain tuning procedure has been followed to ensure fair control parameters selection for the selected techniques. Using challenging test cases, the performance of the selected techniques are assessed experimentally. Comparative experimental results revealed the merits and demerits of individual techniques. Comparative experimental results as presented in this paper have the potential to give some guidance on the selection of the adaptive observer-based sequence extraction techniques for power and energy applications.

Author Contributions: Conceptualization, H.A. and M.B.; methodology, H.A.; software, H.A.; validation, H.A.; formal analysis, H.A. and M.B.; writing-original draft preparation, H.A.; writingreview and editing, M.B.; visualization, H.A. All authors have read and agreed to the published version of the manuscript.

Funding: This research received no external funding.

Institutional Review Board Statement: Not applicable.

Informed Consent Statement: Not applicable.

Data Availability Statement: The data of this study are available from the corresponding author upon reasonable request.

Conflicts of Interest: The authors declare no conflict of interest.

\section{References}

1. Ouchen, S.; Betka, A.; Abdeddaim, S.; Menadi, A. Fuzzy-predictive direct power control implementation of a grid connected photovoltaic system, associated with an active power filter. Energy Convers. Manag. 2016, 122, 515-525. [CrossRef]

2. Safa, A.; Berkouk, E.M.; Messlem, Y.; Gouichiche, A. A robust control algorithm for a multifunctional grid tied inverter to enhance the power quality of a microgrid under unbalanced conditions. Int. J. Electr. Power Energy Syst. 2018, 100, 253-264. [CrossRef]

3. Ngo, T.; Biricik, S.; Basu, M. A Self-tuning Grid Synchronization Method for Active Power Filters. Electr. Power Components Syst. 2016, 44, 1947-1957. [CrossRef]

4. Biricik, S.; Komurcugil, H.; Tuyen, N.D.; Basu, M. Protection of Sensitive Loads Using Sliding Mode Controlled Three-Phase DVR with Adaptive Notch Filter. IEEE Trans. Ind. Electron. 2018, 66, 5465-5475. [CrossRef]

5. Biricik, S.; Redif, S.; Özerdem, Ö.C.; Khadem, S.K.; Basu, M. Real-time control of shunt active power filter under distorted grid voltage and unbalanced load condition using self-tuning filter. IET Power Electron. 2014, 7, 1895-1905. [CrossRef]

6. Biricik, S.; Komurcugil, H.; Ahmed, H.; Babaei, E. Super Twisting Sliding Mode Control of DVR with Frequency-Adaptive Brockett Oscillator. IEEE Trans. Ind. Electron. 2020. [CrossRef]

7. Biricik, S.; Komurcugil, H. Optimized sliding mode control to maximize existence region for single-phase dynamic voltage restorers. IEEE Trans. Ind. Inform. 2016, 12, 1486-1497. [CrossRef]

8. Merai, M.; Naouar, M.W.; Slama-Belkhodja, I.; Monmasson, E. An adaptive PI controller design for DC-link voltage control of single-phase grid-connected converters. IEEE Trans. Ind. Electron. 2019, 66, 6241-6249. [CrossRef]

9. Kukrer, O.; Bayhan, S.; Komurcugil, H. Model-Based Current Control Strategy with Virtual Time Constant for Improved Dynamic Response of Three-Phase Grid-Connected VSI. IEEE Trans. Ind. Electron. 2019, 66, 4156-4165. [CrossRef]

10. Altin, N.; Ozdemir, S.; Komurcugil, H.; Sefa, I. Sliding-mode control in natural frame with reduced number of sensors for three-phase grid-tied LCL-interfaced inverters. IEEE Trans. Ind. Electron. 2018, 66, 2903-2913. [CrossRef]

11. Rahman, M.M.; Hossain, S.; Rezwan, S. Dispatched Power and Unbalanced Operation of a Three-Phase NPC Based DoubleStage Grid Connected PV System. In Proceedings of the 2019 4th International Conference on Electrical Information and Communication Technology (EICT), Khulna, Bangladesh, 20-22 December 2019; IEEE: Piscataway, NJ, USA, 2019 ; pp. 1-6.

12. Chatterjee, A.; Mohanty, K.B. Current control strategies for single phase grid integrated inverters for photovoltaic applications-A review. Renew. Sustain. Energy Rev. 2018, 92, 554-569. [CrossRef]

13. Guerrero-Rodríguez, N.; Rey-Boué, A.B.; Bueno, E.; Ortiz, O.; Reyes-Archundia, E. Synchronization algorithms for grid-connected renewable systems: Overview, tests and comparative analysis. Renew. Sustain. Energy Rev. 2017, 75, 629-643. [CrossRef]

14. Jaalam, N.; Rahim, N.; Bakar, A.; Tan, C.; Haidar, A.M. A comprehensive review of synchronization methods for grid-connected converters of renewable energy source. Renew. Sustain. Energy Rev. 2016, 59, 1471-1481. [CrossRef]

15. Jain, B.; Jain, S.; Nema, R. Control strategies of grid interfaced wind energy conversion system: An overview. Renew. Sustain. Energy Rev. 2015, 47, 983-996. [CrossRef] 
16. Amirat, Y.; Oubrahim, Z.; Ahmed, H.; Benbouzid, M.; Wang, T. Phasor Estimation for Grid Power Monitoring: Least Square vs. Linear Kalman Filter. Energies 2020, 13, 2456. [CrossRef]

17. Ahmed, H.; Biricik, S.; Benbouzid, M. Linear Kalman Filter-Based Grid Synchronization Technique: An Alternative Implementation. IEEE Trans. Ind. Inform. 2020. [CrossRef]

18. Choqueuse, V.; Belouchrani, A.; Auger, F.; Benbouzid, M. Frequency and phasor estimations in three-phase systems: Maximum likelihood algorithms and theoretical performance. IEEE Trans. Smart Grid 2018, 10, 3248-3258. [CrossRef]

19. Mathuria, K.; Hussain, I.; Singh, B.; Kumar, N. A quadrature oscillator-based DT for accurate estimation of fundamental load current for PV system in distribution network. IEEE Trans. Ind. Inform. 2018, 15, 3324-3333. [CrossRef]

20. Ahmed, H.; Biricik, S.; Benbouzid, M. Enhanced Frequency Adaptive Demodulation Technique For Grid-Connected Converters. IEEE Trans. Ind. Electron. 2020. [CrossRef]

21. Chedjara, Z.; Massoum, A.; Massoum, S.; Wira, P.; Safa, A.; Gouichiche, A. A novel robust PLL algorithm applied to the control of a shunt active power filter using a self tuning filter concept. In Proceedings of the 2018 IEEE International Conference on Industrial Technology (ICIT); IEEE: Piscataway, NJ, USA, 2018; pp. 1124-1131.

22. Bechouche, A.; Sediki, H.; Abdeslam, D.O.; Haddad, S. An adaptive neural PLL for grid synchronization. In Proceedings of the IECON 2012-38th Annual Conference on IEEE Industrial Electronics Society; IEEE: Piscataway, NJ, USA, 2012; pp. 4451-4456.

23. Bobrowska-Rafal, M.; Rafal, K.; Jasinski, M.; Kazmierkowski, M. Grid synchronization and symmetrical components extraction with PLL algorithm for grid connected power electronic converters-A review. Bull. Pol. Acad. Sci. Tech. Sci. 2011, 59, $485-497$. [CrossRef]

24. Meral, M.E.; Çelik, D. Benchmarking simulation and theory of various PLLs produce orthogonal signals under abnormal electric grid conditions. Electr. Eng. 2018, 100, 1805-1817. [CrossRef]

25. Meral, M.E.; Çelík, D. A comprehensive survey on control strategies of distributed generation power systems under normal and abnormal conditions. Annu. Rev. Control 2019, 47, 112-132. [CrossRef]

26. Meral, M.E.; Celik, D. DSOGI-PLL based power control method to mitigate control errors under disturbances of grid connected hybrid renewable power systems. Adv. Electr. Electron. Eng. 2018, 16, 81-91. [CrossRef]

27. Ahmed, H.; Pay, M.L.; Benbouzid, M.; Amirat, Y.; Elbouchikhi, E. Gain normalized adaptive observer for three-phase system. Int. J. Electr. Power Energy Syst. 2020, 118, 105821. [CrossRef]

28. Shah, P.; Singh, B. Adaptive Observer Based Control for Roof-top Solar PV System. IEEE Trans. Power Electron. 2020, $35,9402-9415$. [CrossRef]

29. Ahmed, H.; Pay, M.L.; Benbouzid, M.; Amirat, Y.; Elbouchikhi, E. Hybrid estimator-based harmonic robust grid synchronization technique. Electr. Power Syst. Res. 2019, 177, 106013. [CrossRef]

30. Chishti, F.; Murshid, S.; Singh, B. Weak Grid Intertie WEGS With Hybrid Generalized Integrator for Power Quality Improvement. IEEE Trans. Ind. Electron. 2020, 67, 1113-1123. [CrossRef]

31. Bendib, A.; Chouder, A.; Kara, K.; Kherbachi, A.; Barkat, S.; Issa, W. New modeling approach of secondary control layer for autonomous single-phase microgrids. J. Frankl. Inst. 2019, 356, 6842-6874. [CrossRef]

32. Kherbachi, A.; Chouder, A.; Bendib, A.; Kara, K.; Barkat, S. Enhanced structure of second-order generalized integrator frequencylocked loop suitable for DC-offset rejection in single-phase systems. Electr. Power Syst. Res. 2019, 170, 348-357. [CrossRef]

33. Safa, A.; Berkouk, E.M.; Messlem, Y.; Chedjara, Z.; Gouichiche, A. A Pseudo Open Loop Synchronization technique for heavily distorted grid voltage. Electr. Power Syst. Res. 2018, 158, 136-146. [CrossRef]

34. Terriche, Y.; Guerrero, J.M.; Vasquez, J.C. Performance improvement of shunt active power filter based on non-linear least-square approach. Electr. Power Syst. Res. 2018, 160, 44-55. [CrossRef]

35. Hackl, C.M.; Landerer, M. A Unified Method for Online Detection of Phase Variables and Symmetrical Components of Unbalanced Three-Phase Systems with Harmonic Distortion. Energies 2019, 12, 3243. [CrossRef]

36. Ahmed, H.; Benbouzid, M. On the Enhancement of Generalized Integrator-based Adaptive Filter Dynamic Tuning Range. IEEE Trans. Instrum. Meas. 2020, 69, 7449-7457. [CrossRef]

37. Chilipi, R.S.R.; Al Sayari, N.; Al Hosani, K.H.; Beig, A.R. Adaptive Notch Filter-Based Multipurpose Control Scheme for Grid-Interfaced Three-Phase Four-Wire DG Inverter. IEEE Trans. Ind. Appl. 2017, 53, 4015-4027. [CrossRef]

38. Arya, S.R.; Maurya, R.; Naidu, T.A. Amplitude adaptive notch filter with optimized PI gains for mitigation of voltage based power quality problems. CPSS Trans. Power Electron. Appl. 2018, 3, 313-323. [CrossRef]

39. Yazdani, D.; Mojiri, M.; Bakhshai, A.; Joos, G. A fast and accurate synchronization technique for extraction of symmetrical components. IEEE Trans. Power Electron. 2009, 24, 674-684. [CrossRef]

40. Ahmed, H.; Benbouzid, M.; Ahsan, M.; Albarbar, A.; Shahjalal, M. Frequency Adaptive Parameter Estimation of Unbalanced and Distorted Power Grid. IEEE Access 2020, 8, 8512-8519. [CrossRef]

41. Khalil, H.K. Nonlinear Control; Pearson Education North Asia Ltd.: Hong Kong, 2014. 